\title{
Medical Ghostwriting: A University-Sanctioned Sleight of Hand?
}

\author{
Jonathan Leo • Jeffrey R. Lacasse
}

Published online: 31 May 2012

(C) Springer Science + Business Media, LLC 2012

Aside from academic medicine, when most people hear the term "ghostwriter" they think of a paid writer who authored a speech, article, or book without credit. Over the past decade, for virtually every blockbuster medication released, there have been allegations that some of the peer-reviewed papers essential for their commercial success were ghostwritten. The most recent case revolves around two professors of psychiatry at the University of Pennsylvania who were accused of involvement with a ghostwritten paper on the use of the best-selling antidepressant medication, Paxil. Following the charges, the university conducted an internal investigation and, last week, announced that the professors were innocent. The most important ramification of the UPenn investigation, though, is that instead of indicating a vigilant response to ghostwriting, it (perhaps inadvertently) actually sanctions ghostwriting.

As we examined the results of the investigation, we were struck by the fact that the investigative panel seemed to confuse honorary authorship with ghostwriting. To be sure, both are problems in academia, but there are important differences. Honorary authorship consists of someone being placed on the authorship line who did not truly deserve to be listed as an author- often a department head or wellrespected senior researcher in the field. As we have recently argued, ghostwriting is a simpler issue to ascertain, by asking the straightforward question: Was there a writer who contributed significantly to the paper, who was not

Leo and Lacasse have published several articles on medical ghostwriting and the chemical imbalance theory of depression.

J. Leo $(\bowtie) \cdot J$. R. Lacasse

Arizona State University,

Phoenix, AZ 85004, USA

e-mail: Jonathan.leo@Imunet.edu

J. R. Lacasse

e-mail: jeffrey.lacasse@asu.edu listed as an author? If the answer is yes, the paper was ghostwritten. This is not just our perspective. In a recent research article on ghostwriting, the editors of JAMA defined a paper as ghostwritten when, "An individual who was not listed as an author made contributions that merited authorship," or "An unnamed individual participated in writing the article."

The primary conclusions of the University of Pennsylvania investigation did not result from scrutinizing the paper for a ghostwriter, but were instead explanations for why the listed authors deserved to be on the byline. In fact, as reported in the Philadelphia Inquirer, "Susan Phillips, a spokeswoman for the medical school, did not respond to a question about whether the medical writing firm wrote the study or edited the researchers' writing." The final statement concludes that although a medical writer (a subcontractor working for the makers of Paxil) helped write the paper, the listed authors "satisfied all authorship criteria and the publication presented the research findings accurately." Even if the UPenn professor's deserved to be on the byline, if the byline omitted a deserving author then the paper was ghostwritten.

The statement goes on to say that authorship standards have changed in the last decade, and that in 2001, the authors were not breaking any rules. However, regardless of all the other issues involved with this case, this seems to contradict a statement in Senator Charles Grassley's 2010 report on ghostwriting which stated: "Penn Medicine does not use the term 'ghostwriting' in its authorship policies, but stated that it has policies against plagiarism and it considers ghostwriting to be the equivalent of plagiarism." But, regardless of UPenn's past policy, what is of more concern is their new policy, which calls for acknowledging assistance.

According to the results of the recent investigation "... current Perelman School of Medicine policy and journal practice call for acknowledgment of the assistance of a medical writer....." Thus, the University of Pennsylvania 
is setting an institutional norm for authorship where it is appropriate for medical writers to simply be acknowledged at the end of the article, often in small print, for providing "editorial assistance." This despite the fact that it is wellknown that medical writers often write the vast majority of such articles, frequently the first drafts, and are paid employees of the pharmaceutical company with a product to sell. Medical researcher Peter C. Gøtzsche and colleagues note that such acknowledgments are a euphemism for..."XX from Company YY wrote the paper." It would be a simple matter to avoid all this by simply listing medical writers as authors, thus presenting authorship transparently (a plan advocated by one notable medical writer), while we can think of only one reason not to do so: Allowing the listing of an author in the acknowledgement section is an academic sleight of hand that obscures a conflict-of-interest from the readers.

Some might say that listing authors in the acknowledgement section is full disclosure, but "editorial assistants" are not listed in pub med, are not listed in the abstract, they are not cited, and they are not called by the media to talk about the importance of a study. And, other than minimizing the company's role in the study, there seems to be no good reason for not giving them their due credit. In a sense, the published paper also carries the endorsement of the university employing the named authors. The only parties to benefit from re-defining authorship in this way are pharmaceutical companies, who we know from their own internal documents, see the peer-reviewed literature primarily as a venue for promoting their products. Forest Pharmaceutical's marketing plan for the antidepressant Lexapro states: "Bylined articles will allows us to fold Lexapro's message into articles into depression, anxiety, and comorbidity developed by (or ghostwritten) for thought leaders."

Usually the acknowledgment section is reserved for those who did not rise to the level of author. Listing the primary authors in the acknowledgements rather than the byline represents a fundamental change in how science operates. Importantly, the usual explanations offered to explain why papers that omit industry-funded authors from the byline are not ghostwritten such as the work was peer-reviewed; the research is accurate; the lead author is a great scientist; the average reader cannot detect bias in the paper, have nothing to do whatsoever with the issue. Imagine a Newsweek cover story praising Toyota whose first draft was written by a Toyota employee who was not included in the byline but instead was thanked for "editorial assistance." The general public would hardly stand for this behavior in the popular press. Likewise, it would be hard to imagine the Newsweek editors using any of the excuses used by academics to defend this practice. Most people from outside the halls of academia would probably be surprised that the idea of not listing all the authors on a paper's byline is even debatable.
If the byline, which is usually the second line of a paper and right underneath the title, is not accurate, why should readers trust the rest of the paper?

Although we have no doubt that many faculty at UPenn would like to eliminate ghostwriting, this is not actually reflected in their policy. The most famous ghostwritten paper in the peer-reviewed literature is Study 329, a failed pediatric study of Paxil that selectively reported positive results while downplaying the adverse effects. In the scientific paper resulting from Study 329, medical writer Sally Laden was acknowledged for editorial assistance, even though she wrote the first draft of the paper, and was involved in all the subsequent drafts. Yet, although this study is openly referred to in the peer-reviewed literature as being ghostwritten, according to the UPenn's investigational panel, it would not be considered ghostwritten. UPenn's policy (and that of many other academic medical centers) of allowing papers with invisible authors is nothing but an endorsement of ghostwriting.

This leads to many implications for both academic research and the education of aspiring health professionals such as physicians and nurses. In terms of research, it is obvious that there are an undetermined (but large) number of ghostwritten papers in the peer-reviewed medical literature. Systematic reviews and meta-analyses of topics where pharmaceutical companies have a stake need to be reexamined with this in mind. There is currently no mechanism in place for handling known ghostwritten papers, and many continue to be cited favorably. Academia might also rethink the perception of pharmaceutical industry-affiliated professors far from retirement who already have nearly 1,000 publications on their curricula vitae. Rather than regarding them as luminaries, we might wonder how many of their publications are ghostwritten, especially in the 1990s and 2000s when, according to the UPenn investigation, policies were not in place to prevent ghostwriting.

Critics of ghostwriting are not calling for a ban on joint research projects between company employees and university researchers, nor on the use of medical writers, but are instead simply asking for accurate bylines. When a medical writer deserves to be called an author they should be listed on the byline. And it's not as if this approach is unheard of. Some companies such as Eli Lilly frequently do just this, listing the company employees as authors. Likewise, accurate bylines are hardly the sole solution to all the problems with conflicts of interest in medicine, but they are a fairly simple step in the right direction. One can only speculate, but given accurate authorship bylines would the medical community have approached the clinical trial literature supporting the use of the blockbuster medications with just a bit more skepticism?

The significance of the shift in authorship guidelines that UPenn is describing has yet to be fully appreciated by the 
wider academic community, and many academics, along with the general public, will find it astonishing, or at least perplexing. To retain the credibility and the mission of rigorous scientific investigation, academic medicine must take a strong stance against ghostwriting, a stance that is consistent with authorship norms across the university.

\section{Further Reading}

Healy D, Cattell D. 2003. Interface between authorship, industry and science in the domain of therapeutics. British Journal of Psychiatry [Internet], Jul 1 [cited 2011 Feb 2];183(1), 22-27. Available from: http://bjp.rcpsych.org/cgi/content/abstract/183/1/22

Jureidini, J. N., McHenry, L. B., \& Mansfield, P. R. 2008. Clinical trials and drug promotion: Selective reporting of study 329. Intern Journ Risk and Safety in Medicine, 20(1-2), 73-81.

Lacasse JR, Leo J. 2010. Ghostwriting at elite academic medical centers in the United States. PLoS Medicine [Internet], Feb 2;7
(2), e1000230. Available from: http://www.plosmedicine.org/article/info\%3Adoi\%2F10.1371\%2Fjournal.pmed.10002

Matheson, A. 2011. How industry uses the ICMJE guidelines to manipulate authorship - and how they should be revised. PLoS Medicine [Internet], 8(8), e1001072. doi:10.1371/journal.pmed.1001072. Available from: http://www.plosmedicine.org/article/ info\%3Adoi\%2F10.1371\%2Fjournal.pmed.1001072

Moffatt B, Elliott C. 2007. Ghost marketing: Pharmaceutical companies and ghostwritten journal articles. Perspectives in Biology and Medicine [Internet], [cited 2011 Feb 2];50(1), 18-31. Available from: http://www.webcitation.org/5wJIVyU8j

Sismondo S. 2007. Ghost management: How much of the medical literature is shaped behind the scenes by the pharmaceutical industry? PLoS Medicine [Internet]. Sep [cited 2011 Feb 2];4(9), e286. Available from: http://dx.doi.org/10.1371/journal.pmed.0040286

Jonathan Leo is Professor of Neuroanatomy at Lincoln Memorial University in Harrogate TN. Jeffrey Lacasse is Assistant Professor of Social Work at Arizona State University. 